\title{
CCNE1 amplification is associated with aggressive potential in endometrioid endometrial carcinomas
}

\author{
KENTARO NAKAYAMA $^{1 *}$, MOHAMMED TANJIMUR RAHMAN $^{1 *}$, MUNMUN RAHMAN $^{1}$, \\ KOHEI NAKAMURA ${ }^{1}$, MASAKO ISHIKAWA ${ }^{1}$, HIROSHI KATAGIRI ${ }^{1}$, EMI SATO $^{1}$, \\ TOMOKA ISHIBASHI $^{1}$, KOUJI IIDA ${ }^{1}$, NORIYUKI ISHIKAWA ${ }^{2}$ and SATORU KYO ${ }^{1}$
}

Departments of ${ }^{1}$ Obstetrics and Gynecology, ${ }^{2}$ Organ Pathology, Shimane University School of Medicine, Izumo 6938501, Japan

Received October 6, 2015; Accepted November 8, 2015

DOI: $10.3892 /$ ijo.2015.3268

\begin{abstract}
The clinicopathological significance of amplification was investigated of the gene encoding cyclin E (CCNEI) and we assessed whether CCNE1 was a potential target in endometrioid endometrial carcinomas. CCNE1 amplification and CCNE1 or F-box and WD repeat domain-containing 7 (FBXW7) expression in endometrial endometrioid carcinoma was assessed by immunohistochemistry and fluorescence in situ hybridization. CCNE1 knockdown by small interfering RNA (siRNA) was used to assess the CCNEI function. The results showed that $C C N E 1$ amplification was present in 9 (8.3\%) of 108 endometrial carcinomas. CCNE1 amplification was correlated with high histological grade (Grade 3; $\mathrm{P}=0.0087)$ and lymphovascular space invasion $(\mathrm{P}=0.0258)$. No significant association was observed between $C C N E 1$ amplification and FIGO stage $(\mathrm{P}=0.851)$, lymph node metastasis $(\mathrm{P}=0.078)$, body mass index $(\mathrm{P}=0.265)$, deep myometrial invasion $(\mathrm{P}=0.256)$, menopausal status $(\mathrm{P}=0.289)$ or patient age $(\mathrm{P}=0.0817)$. CCNE1 amplification was significantly correlated with shorter progression-free and overall survival $(\mathrm{P}=0.0081$ and 0.0073 , respectively). CCNE1 protein expression or loss of FBXW7 expression in endometrial endometrioid carcinoma tended to be correlated with shorter progression-free and overall survival; however, this difference was not statistically significant. Multivariate analysis showed that CCNEI amplification was an independent prognostic factor for overall survival but not for progression-free survival $(\mathrm{P}=0.0454$ and 0.2175 , respectively). Profound growth inhibition was observed in siRNA-transfected cancer cells with endogenous CCNE1 overexpression compared with that in cancer cells having low
\end{abstract}

Correspondence to: Dr Kentaro Nakayama, Shimane University School of Medicine, Enyacho 89-1, Izumo, Shimane 6938501, Japan E-mail:kn88@med.shimane-u.ac.jp

*Contributed equally

Key words: cyclin E1, gene amplification, F-box and WD repeat domain-containing 7, endometrial carcinoma
CCNE1 expression. CCNE1 amplification was independent of p53, HER2, MLH1 and ARID1A expression but dependent on PTEN expression in endometrial carcinomas. These findings indicated that $C C N E 1$ amplification was critical for the survival of endometrial endometrioid carcinomas. Furthermore, the effects of CCNE1 knockdown were dependent on the CCNE1 expression status, suggesting that CCNE1-targeted therapy may be beneficial for patients with endometrial endometrioid carcinoma having $C C N E 1$ amplification.

\section{Introduction}

Endometrial carcinomas are the most common gynecological malignancies and the fifth most common cancer in women worldwide (1). Endometrial carcinomas can be subdivided into two main categories: type I, estrogen-dependent endometrioid carcinoma; and type II, estrogen-independent non-endometrioid carcinoma $(2,3)$.

Studies have shown that exposure to high levels of estrogen is an important risk factor for endometrial carcinomas (2). Endometrioid carcinoma represents $\sim 80-85 \%$ of all endometrial malignancies. Previous studies have identified various genetic alterations in endometrioid carcinoma, including silencing of the phosphatase and tensin homolog deleted on chromosome 10 (PTEN) gene, microsatellite instability (MSI), and mutations in K-ras and/or PIK3CA (3-6).

While the majority of endometrial carcinomas are diagnosed at an early stage, resulting in a favorable prognosis, women diagnosed with advanced or recurrent disease have much lower survival rates and limited adjuvant treatment options. Over the past few decades, survival rates in patients with advanced disease have not improved sufficiently.

Our recent genome-wide sequencing analyses of all exons and transcriptomes in uterine serous carcinomas (USCs) showed that approximately half of all USC cases harbor either somatic mutations in F-box and WD repeat domaincontaining 7 (FBXW7) or gene amplification in CCNE1 (encoding cyclin E1) (7). The cyclin E-FBXW7 pathway is thought to be one of the most important pathways in USC development (7).

FBXW7 is the substrate recognition component of the Skp1-Cul1-F-box (SCF) ubiquitin-ligase and is located within $4 \mathrm{q} 32$, a chromosomal region that is commonly deleted in 
cancers (8-10). FBXW7 acts as a tumor suppressor by targeting several oncogenic regulators of proliferation, growth and apoptosis for proteasomal degradation. These include cyclin $\mathrm{E}$, c-MYC, Notch and MCL1 (11-14). Furthermore, lower expression of FBXW7 contributes to lymph node metastasis, tumor size and poor prognosis in gastric cancer (15).

DNA copy number alterations, including amplification, deletion, and aneuploidy in chromosomes, are the hallmarks of neoplasia (16). Amplification of chromosomal regions plays a critical role in tumor development. Increases in the copy numbers of oncogenes promote initiation and progression of a variety of solid tumors, whereas amplification of genes that modify or detoxify chemotherapeutic agents can lead to drug resistance and is associated with tumor recurrence $(17,18)$. Well-known amplified oncogenes include c-myc, ERBB2, EGFR, AKT2, CCND1 and CCNE1. Studies on these genes have not only provided new insights into the mechanisms of cancer development but also revealed significant translational implications. For example, ERBB2 and EGFR are molecular targets of the humanized antibodies trastuzumab (Herceptin) and matuzumab, respectively, which are used in the treatment of breast and lung cancer.

Although CCNE1 amplifications and FBXW7 mutations are frequently present in serous-type endometrial cancer, the clinicopathological and prognostic roles of these modifications in endometrioid-type endometrial cancer are still unclear. Inactivating mutations in tumor suppressors could participate not only in tumor initiation, but also in tumor progression and response to therapy. In the present study, we examined the prognostic and clinicopathological significance of CCNE1 amplification/expression and loss of FBXW7 expression in endometrial carcinoma by investigating the relationship between CCNE1 amplification/expression or FBXW7 expression and various clinicopathological variables in endometrial carcinoma. In addition, we compared phenotypes in cultured endometrial carcinoma cells with variations in CCNE1 expression levels after transfection with small interfering RNA (siRNA) targeting CCNE1.

\section{Materials and methods}

Tissue samples. Formalin-fixed, paraffin-embedded tissue samples from 108 endometrioid-type endometrial carcinomas were used in this study. Samples were obtained from the Department of Obstetrics and Gynecology at the Shimane University Hospital. Diagnosis was based on conventional morphological examination of hematoxylin and eosin (H\&E)stained sections. Cancer patients had received appropriate therapy at Shimane University Hospital between January 1998 and August 2010. Acquisition of tissue specimens and clinical information was approved by the institutional review board of Shimane University. Endometrial carcinomas were classified according to the surgical staging system of the International Federation of Gynecology and Obstetrics (FIGO). Invasive carcinomas were divided by stage, with 76 patients having stage I disease, 9 patients having stage II disease, 17 patients having stage III disease, and 6 patients having stage IV disease. All tumors were classified histologically according to the World Health Organization criteria. The median patient age was 60 years (range, 26-85 years). Primary surgeries included total hysterectomy in 39 patients, modified radical hysterectomy in 60 patients and radical hysterectomy in 9 patients. Systemic retroperitoneal lymphadenectomy was performed in $\sim 90 \%$ of patients. Patients with risk factors for recurrence (e.g., deep myometrial invasion, cervical invasion, lymph node metastasis, lymphovascular space invasion and positive peritoneal cytology) underwent postoperative adjuvant external radiotherapy and/or 4-6 cycles of CAP therapy (cisplatin $100 \mathrm{mg} / \mathrm{m}^{2}$, doxorubicin $40 \mathrm{mg} / \mathrm{m}^{2}$, cyclophosphamide $500 \mathrm{mg} / \mathrm{m}^{2}$ ) or TC therapy (paclitaxel $175 \mathrm{mg} / \mathrm{m}^{2}$, carboplatin according to Chatelut's formula - area under the curve $=5 \mathrm{mg} / \mathrm{m}^{2}$ ). All 108 patients were included in the survival analysis. The follow-up period ranged from 5 to 139 months, with a median of 52 months.

Fluorescence in situ hybridization (FISH). BAC clones (RP11345J21 and CTD-3005A16) containing the genomic sequences of the 19q12 amplicon at 15.00-15.25 Mb were purchased from Bacpac Resources Center (Children's Hospital, Oakland, CA, USA) and Invitrogen (Carlsbad, CA, USA). Bac clones located at Chr2q11.2 (e.g., RP11-127K18 and RP11-629A22) or at Ch19P12 (CTD-2518018) were used to generate reference probes.

RP11-127K18, RP11-629A22 and CTD-2518018 were labeled by nick translation with biotin-dUTP; RP11-345J21 and CTD-3005A16 were labeled similarly with digoxigenindUTP. To detect biotin-labeled and digoxigenin-labeled signals, slides were first incubated with FITC-avidin (Vector Laboratories, Burlingame, CA, USA) and an anti-digoxigenin mouse antibody (Roche Molecular Biochemicals, Mannheim, Germany). Slides were subsequently incubated with a biotinylated anti-avidin antibody (Vector Laboratories) and tetramethylrhodamine B isothiocyanate (TRITC)-conjugated rabbit anti-mouse antibodies (Sigma, St. Louis, MO, USA). The final incubation was with FITC-avidin and TRITC-conjugated goat anti-rabbit antibodies (Sigma). Slides were counterstained with 4',6'-diamidino-2-phenylindole stain (Sigma).

Fluorescence in situ hybridization (FISH) signals were evaluated with an Olympus fluorescence microscope BX41 (Olympus, Tokyo, Japan) by two individuals who were blinded to the treatment history of each patient. Separate narrow band pass filters were used for detection of tetramethylrhodamine B isothiocyanate, FITC, and 4',6'-diamidino-2-phenylindole staining signals. Using a x60 objective lens, 100 tumor cells were examined for each specimen, and the numbers of fluorescent signals within tumor cells from the CCNE1 gene BAC probe and chromosome $2 \mathrm{q} 11.2$ or $19 \mathrm{p} 12$ reference BAC probe were recorded. Amplification of $C C N E 1$ was defined as the ratio of CCNE1 BAC probe signals to chromosome 2 or chromosome 19 centromeric reference BAC probe signals of 2:1 or more.

Immunohistochemistry. Paraffin-embedded tissues were organized into tissue microarrays, which were made by removing tumor cores ( $3 \mathrm{~mm}$ in diameter) from each block. Selection of the area to core was made by a gynecologic oncologist (K.N.) and pathology technician (K.I.) and was based on a review of the H\&E slides.

Expression of CCNE1 and FBXW7 was assessed by immunohistochemistry and/or western blot analysis. The antibodies 
used in the present study were mouse monoclonal antibodies targeting CCNE1 (Zymed Laboratories-Invitrogen, Carlsbad, CA, USA) and mouse monoclonal antibodies targeting FBXW7 (Abcam, Cambridge, MA, USA). Immunohistochemistry studies for CCNE1 and FBXW7 were performed on tissue microarrays at a dilution of 1:250 or 1:50 followed by detection with the EnVision+ system using the peroxidase method (Dako, Carpinteria, CA, USA). After antigen retrieval in sodium citrate buffer, slides were incubated with antibodies overnight at $4^{\circ} \mathrm{C}$. Slides for all samples were evaluated with a light microscope by two researchers; the researchers were blinded to the clinicopathological factors. The antibody staining intensity was then analyzed in glands using the HSCORE (19). The modified HSCORE was calculated as follows: HSCORE $=\Sigma \mathrm{P}_{i}(i)$, where $i$ is the intensity of staining ( 0 , undetectable, 1 , weakly positive, 2 , moderately positive, 3 , intensely positive) and $\mathrm{P}_{i}$ is a score based on the percentage of stained cells for each intensity, varying from 0 to $100 \%$.

Immunohistochemistry methods for ARID1A, PTEN, MLH1, p53, HER2 ER and progesterone receptor (PR), as well as evaluation criteria, have been previously described $(20,21)$.

Cell culture and cell lines. The human endometrial carcinoma cell lines HEC251 (endometrioid carcinoma), HEC50B (endometrioid carcinoma G3), and HEC108 (endometrioid carcinoma) were obtained from the Japanese Health Science Research Resources Bank (Osaka, Japan). HHUA (endometrioid carcinoma) and JHUC-1 (endometrial carcinosarcoma) cells were obtained from Riken Bioresource Center (Ibaragi, Japan).

Western blot analysis. Cell lysates were prepared by dissolving cell pellets in Laemmli sample buffer (Bio-Rad Laboratories, Hercules, CA, USA) supplemented with $5 \%$ beta-mercaptoethanol (Sigma). Western blot analysis was performed on endometrial carcinoma cell lines, including HEC251, HEC50B, HHUA, JHUC-1 and HEC108 cells. Similar amounts of total protein from each lysate were loaded and separated on $10 \%$ Tris-Glycine-SDS polyacrylamide gels (Novex, San Diego, CA, USA) and electroblotted to Millipore Immobilon-P polyvinylidene difluoride membranes (Millipore, Bedford, MA, USA). Membranes were probed with anti-CCNE1 antibodies (1:1,000 dilution; Zymed Laboratories-Invitrogen) followed by peroxidase-conjugated anti-mouse or anti-rabbit immunoglobulin (1:20,000 dilution). The same membrane was probed with antibodies targeting GAPDH (1:10,000 dilution; Cell Signaling Technology, Beverly, MA, USA) as a loading control. Western blots were developed by chemiluminescence (Pierce, Rockford, IL, USA).

siRNA-mediated knockdown of CCNE1 gene expression. Two siRNAs that targeted $C C N E 1$ were designed with the following sense sequences: UCAGUUGACAGUGUACAAUGCCUTT and UGACUUACAUGAAGUGCUACUGCCG. Control siRNA (luciferase siRNA) was purchased from Integrated DNA Technologies (Coralville, IA, USA). Cells were seeded onto 96-well plates and transfected with siRNAs using Oligofectamine (Invitrogen). Cell numbers were determined indirectly using the 3-(4,5-dimethylthiazol-2-yl)-2,5-diphenyltetrazolium bromide (MTT) assay $72 \mathrm{~h}$ after transfection (22).
Cell proliferation assay. Cells were seeded in 96-well plates at a density of 3,000 cells/well. Cell numbers were determined indirectly using MTT assays (22). Data were expressed as the mean \pm standard deviation (SD) from triplicate determinations.

Statistical methods for clinical correlations. Overall survival was calculated from the date of diagnosis to the date of death or last follow-up. Patients with and without CCNE1 or FBXW7 expression or CCNE1-amplified tumors had similar ages and performance status distributions. Statistical significance was determined by the log-rank test. Data were censored when patients were lost to follow-up.

\section{Results}

Relationships among CCNE1 amplification, CCNE1 protein expression, FBXW7 protein expression and clinicopathological factors. As shown in Table I, the frequency of CCNE1 amplification in high-grade carcinomas (G3; 20.6\%: 6/29) was significantly higher than that in low-grade tumors (G1, G2; 3.8\%: 3/79; $\mathrm{P}=0.0087)$. CCNE1 amplification was also correlated with lymphovascular space invasion $(\mathrm{P}=0.0258)$. There were no significant correlations between $C C N E 1$ amplification and FIGO stage $(\mathrm{P}=0.851)$, lymph node metastasis $(\mathrm{P}=0.078)$, patient age $(\mathrm{P}=0.0817)$, body mass index $(\mathrm{P}=0.265)$, deep myometrial invasion $(\mathrm{P}=0.256)$ or menopausal status $(\mathrm{P}=0.289$; Table I).

Patients were stratified into one of two groups depending on the median CCNE1 immunohistochemical HSCORE. The relationships between CCNE1 protein expression and clinicopathological factors are shown in Table I. CCNE1 protein expression was significantly correlated with highgrade carcinoma $(\mathrm{P}=0.0169)$, lymphovascular space invasion $(\mathrm{P}=0.0195)$, postmenopausal status $(\mathrm{P}=0.0281)$, and patient age $(\mathrm{P}=0.0209)$. There was no significant correlation between CCNE1 protein expression and FIGO stage $(\mathrm{P}=0.0601)$, body mass index $(\mathrm{P}=0.5148)$, lymph node metastasis $(\mathrm{P}=0.7808)$, or deep myometrial invasion $(\mathrm{P}=0.1651)$.

Negative expression of FBXW7 (FBXW7 immunohistochemical HSCORE $=0)$ was observed in $62 \%(63 / 101)$ of the analyzed tumors. Patients were stratified into one of two groups depending on the status of the negative FBXW7 immunohistochemical HSCORE. The relationships between FBXW7 protein expression and clinicopathological factors are shown in Table I. Negative FBXW7 protein expression was significantly correlated with deep myometrial invasion $(\mathrm{P}=0.0144)$, lymphovascular space invasion $(\mathrm{P}=0.0414)$ and patient age $(\mathrm{P}=0.038)$. There was no significant correlation between negative FBXW7 protein expression and FIGO stage $(\mathrm{P}=0.2439)$, tumor grade $(\mathrm{P}=0.228)$, lymph node metastasis $(\mathrm{P}=0.4805)$, postmenopausal status $(\mathrm{P}=0.882)$ or body mass index $(\mathrm{P}=0.162)$.

Effects of CCNE1 amplification or CCNE1 protein expression on progression-free survival. Next, we evaluated the prognostic relevance of CCNE1 amplification or CCNE1 protein expression in terms of progression-free survival. Kaplan-Meier estimates of progression-free/overall survival are plotted in Fig. 2. Amplification of CCNE1 correlated with shorter progression-free survival in patients with endometrial 
Table I. Association between CCNE1 gene amplification and clinocopathological factors in patients with endometrial cancer.

\begin{tabular}{|c|c|c|c|c|c|c|c|c|c|c|}
\hline \multirow[b]{2}{*}{ Factors } & \multirow[b]{2}{*}{ Patients } & \multicolumn{2}{|c|}{$\begin{array}{l}C C N E 1 \text { gene } \\
\text { amplification }\end{array}$} & \multirow[b]{2}{*}{ P-value } & \multicolumn{2}{|c|}{$\begin{array}{l}\text { CCNE1 } \\
\text { protein } \\
\text { expression }\end{array}$} & \multirow[b]{2}{*}{ P-value } & \multicolumn{2}{|c|}{$\begin{array}{l}\text { FBXW7 protein } \\
\text { expression }\end{array}$} & \multirow[b]{2}{*}{ P-value } \\
\hline & & Negative & Positive & & Low & High & & Negative & Positive & \\
\hline \multicolumn{11}{|l|}{ FIGO stage } \\
\hline I & 75 & 69 & 6 & 0.851 & 42 & 33 & 0.0601 & 41 & 34 & 0.2439 \\
\hline II, III, IV & 33 & 30 & 3 & & 12 & 21 & & 22 & 11 & \\
\hline \multicolumn{11}{|l|}{ Grade } \\
\hline $\mathrm{G} 1, \mathrm{G} 2$ & 79 & 76 & 3 & 0.0087 & 45 & 34 & 0.0169 & 45 & 34 & 0.228 \\
\hline G3 & 29 & 23 & 6 & & 9 & 20 & & 18 & 11 & \\
\hline \multicolumn{11}{|c|}{ Lymph node metastasis } \\
\hline Negative & 93 & 87 & 6 & 0.078 & 47 & 46 & 0.7808 & 53 & 40 & 0.4805 \\
\hline Positive & 15 & 12 & 3 & & 7 & 8 & & 10 & 5 & \\
\hline \multicolumn{11}{|c|}{ Depth (myometrial invasion) } \\
\hline $\mathrm{a}, \mathrm{b}$ & 67 & 63 & 4 & 0.256 & 37 & 30 & 0.1651 & 33 & 34 & 0.0144 \\
\hline $\mathrm{c}$ & 41 & 36 & 5 & & 17 & 24 & & 30 & 11 & \\
\hline \multicolumn{11}{|c|}{$\begin{array}{l}\text { Lymphovascular space } \\
\text { invasion }\end{array}$} \\
\hline Negative & 62 & 60 & 7 & 0.0258 & 37 & 25 & 0.0195 & 31 & 31 & 0.0414 \\
\hline Positive & 46 & 39 & 7 & & 17 & 29 & & 32 & 14 & \\
\hline \multicolumn{11}{|l|}{ Menopause } \\
\hline Peri, pre & 28 & 27 & 1 & 0.289 & 19 & 9 & 0.0281 & 16 & 12 & 0.882 \\
\hline Post & 80 & 72 & 8 & & 35 & 45 & & 47 & 33 & \\
\hline \multicolumn{11}{|c|}{ Body mass index } \\
\hline$<25$ & 79 & 71 & 8 & 0.265 & 41 & 38 & 0.5148 & 47 & 32 & 0.162 \\
\hline$\geq 25$ & 29 & 28 & 1 & & 13 & 16 & & 16 & 13 & \\
\hline \multicolumn{11}{|l|}{ Age (years) } \\
\hline$<60$ & 54 & 52 & 2 & 0.0817 & 33 & 21 & 0.0209 & 31 & 23 & 0.038 \\
\hline$\geq 60$ & 54 & 47 & 7 & & 21 & 33 & & 32 & 22 & \\
\hline
\end{tabular}

endometrioid carcinomas. A total of 108 patients were diagnosed at stages I-IV. Among them, the 9 patients with CCNEI amplifications had shorter progression-free survival than those without gene amplification ( $\mathrm{P}=0.0081$; log-rank test; Fig. 2A). Univariate analysis demonstrated that deep myometrial invasion ( $\mathrm{P}=0.0008$; log-rank test), high-grade histological subtype $(\mathrm{P}<0.0001$; log-rank test), lymph node metastasis $(\mathrm{P}<0.0001$; log-rank test), lymphovascular space invasion $(\mathrm{P}=0.001$; logrank test) and $C C N E 1$ amplification $(\mathrm{P}=0.0081$; log-rank test $)$ correlated with shorter progression-free survival. CCNE1 HSCORE tended to influence progression-free survival, but this relationship was not statistically significant $(\mathrm{P}=0.3148$; Fig. 2B). We excluded lymph node metastasis and lymphovascular space invasion from multivariate analysis because these two factors are similar events in the clinical setting and to prevent any issues with statistical analyses. Therefore, for multivariate analysis, we chose FIGO stage, tumor grade, depth of myometrial invasion and CCNEI amplification. When the data were stratified for multivariate analysis, high-grade histological subtype and deep myometrial invasion remained significantly associated with shorter progression-free survival $(\mathrm{P}=0.0003$ and $\mathrm{P}=0.0142$, respectively; Table II).

Effects of CCNE1 amplification or CCNE1 protein expression on overall survival. Next, we examined the prognostic relevance of CCNE1 amplification or CCNE1 protein expression in terms of overall survival. Kaplan-Meier estimates of progression-free/overall survival are plotted in Fig. 3. Amplification of CCNE1 correlated with shorter overall survival in patients with endometrial endometrioid carcinomas treated. A total of 108 patients were diagnosed at stages I-IV. Among them, the 9 patients with $C C N E 1$ amplification had shorter overall survival than those without $\mathrm{CCNE} 1$ amplification $(\mathrm{P}=0.0073$; log-rank test; Fig. 2C). Univariate analysis demonstrated that FIGO stage III or IV ( $\mathrm{P}=0.035$; log-rank test), highgrade histological subtype $(\mathrm{P}<0.0001 ; \log$-rank test $)$, deep myometrial invasion ( $\mathrm{P}=0.0008$; log-rank test), lymph node metastasis $(\mathrm{P}<0.0001$; log-rank test $)$, lymphovascular space 
Table II. Univariate analysis of progression-free prognostic factors in patients with endometrial carcinoma.

\begin{tabular}{lccccc}
\hline & Univariate & & Multivariate \\
Factors & Patients & $\begin{array}{c}\text { hazard ratio } \\
\text { hazard ratio }\end{array} \quad 95 \%$ CI & P-value & P-value
\end{tabular}

FIGO stage

I

II, III, IV

Grade

$\mathrm{G} 1, \mathrm{G} 2$

G3

Lymph node metastasis

Negative

Positive

Depth (myometrial invasion)

$\begin{array}{ll}\mathrm{a}, \mathrm{b} & 67 \\ \mathrm{c} & 41\end{array}$

Lymphovascular space invasion

Negative

Positive

Menopause

Peri, pre

Post

Body mass index

$<25$

$\geq 25$

Age (years)

$<60$

$\geq 60$

CCNE1 FISH

Normal

Amp

CCNE1 immunostaing

Low

High

FBXW7 immunostaing

Positive

Negative

75

33

79

29

93

15

62

46

28

80

79

29

54

54

$$
9
$$

99

54

54

45

63
27.1

$6.0-122.1$

$<0.0001$

18.2

$3.8-86.8$

0.0003

9.7

3.4-27.7

$<0.0001$

NA

NA

NA

8.824

2.5-31.4 0.0008

5.3

$1.4-19.8$

0.0142

30.5

4.0-233.6

0.001

NA

NA

NA

5.5

$0.7-42.0$

0.0991

NA

NA

NA

1.1

$0.3-3.5$

0.8597

NA

NA

NA

2.9

$0.9-9.1$

0.069

NA

NA

NA

4.7

$1.5-14.8$

0.0081

2.2

0.6-7.4

0.2175

1.7

$0.6-4.8$

0.3148

NA

NA

NA

1.9

0.6-5.5

0.2637

NA

NA

NA invasion $(\mathrm{P}=0.0011$; log-rank test $)$, and $C C N E 1$ amplification $(\mathrm{P}=0.0073$; log-rank test) were correlated with shorter overall survival. High CCNE1 HSCOREs tended to be associated with poor overall survival; however, this relationship was not significant ( $\mathrm{P}=0.2205$; Fig. 2D). As described in the previous section, lymph node metastasis and lymphovascular space invasion were excluded from multivariate analysis. Therefore, for multivariate analysis, we chose FIGO stage, tumor grade, depth of myometrial invasion and CCNE1 amplification. When data were stratified for multivariate analysis, high-grade subtype, deep myometrial invasion and CCNE1 amplification remained significantly associated with shorter overall survival ( $\mathrm{P}=0.0006, \mathrm{P}=0.0252$ and $\mathrm{P}=0.0454$, respectively; Table III).

Effects of FBXW7 protein expression on disease-free/overall survival. Because FBXW7 is the substrate recognition component of the Skp1-Cul1-F-box (SCF) ubiquitin-ligase, which targets CCNE1, we next analyzed whether loss of FBXW7 immunostaining was related to poor survival and whether there was a positive relationship between loss of FBXW7 protein expression and CCNE1 protein expression. Immunoreactivity for FBXW7 was detected in tumor cell 
Table III. Univariate analysis of overall prognostic factors in patients with endometrial carcinoma.

\begin{tabular}{|c|c|c|c|c|c|}
\hline Factors & Patients & $\begin{array}{l}\text { Univariate } \\
\text { hazard ratio }\end{array}$ & $95 \% \mathrm{CI}$ & P-value & $\begin{array}{l}\text { Multivariate } \\
\text { hazard ratio }\end{array}$ \\
\hline
\end{tabular}

FIGO stage

I

II, III, IV

Grade

$\mathrm{G} 1, \mathrm{G} 2$

G3

Lymph-node metastasis

Negative

Positive

Depth (myometrial invasion)

$$
\mathrm{a}, \mathrm{b}
$$

c

Lymphovascular space invasion

\section{Negative}

Positive

Menopause

Peri, pre

Post

Body mass index

$<25$

$\geq 25$

Age (years)

$<60$

$\geq 60$

CCNE1 FISH

Normal

Amp

CCNE1 immunostaing

$\begin{array}{ll}\text { Low } & 54 \\ \text { High } & 54\end{array}$

FBXW7 immunostaing

Positive

Negative

33

79

29

93

15

67

41

62

46

28

80

54

54

9

99

54

54

45

63
26.7

5.9-120.3

$<0.0001$

15.7

3.2-76.1

0.0006

11.7

4.0-33.8

$<0.0001$

NA

NA

NA

8.8

2.5-31.4 0.0008

4.6

1.2-17.4

0.0252

29.4

3.9-224.3

0.0011

NA

NA

NA

5.3

$0.7-40.2$

0.1081

NA

NA

NA

1.1

0.4-3.6

0.883

NA

NA

NA

2.9

$0.9-9.1$

0.067

NA

NA

NA

4.8

$1.5-15.2 \quad 0.0073$

3.8

1.0-14.0

0.0454

1.9

0.7-5.4

0.2205

NA

NA

NA

1.9

0.7-5.7

0.2307

NA

NA

NA nuclei (Fig. 1). The mean HSCORE of FBXW7 was 46 (range, 0-300). The CCNE1 immunohistochemical HSCORE was not correlated with the FBXW7 immunohistochemical HSCORE (data not shown). Negative expression of FBXW7 (FBXW7 immunohistochemical HSCORE = 0) was observed in 58.3\% $(63 / 108)$ of the analyzed tumors. Patients were stratified into one of two groups depending on the status of negative FBXW7 immunohistochemical HSCORE. Kaplan-Meier estimates of progression-free/overall survival are plotted in Fig. 2. Negative FBXW7 expression tended to correlate with shorter overall/progression-free survival in patients with endometrial endometrioid carcinoma; however, this relationship was not statistically significant (Fig. 2E andF).

Relationship between CCNE1 amplification and PTEN, p53, HER2, MLH1 or ARID1A expression in endometrial endometrioid carcinoma. We previously performed immunohistochemical analysis to evaluate the status of PTEN, p53, HER2, MLH1 and ARID1A expression among analyzed tumor samples (20). We then investigated the correlations between the expression of these molecules and CCNE1 amplification (Table IV). No significant correlations were observed between 


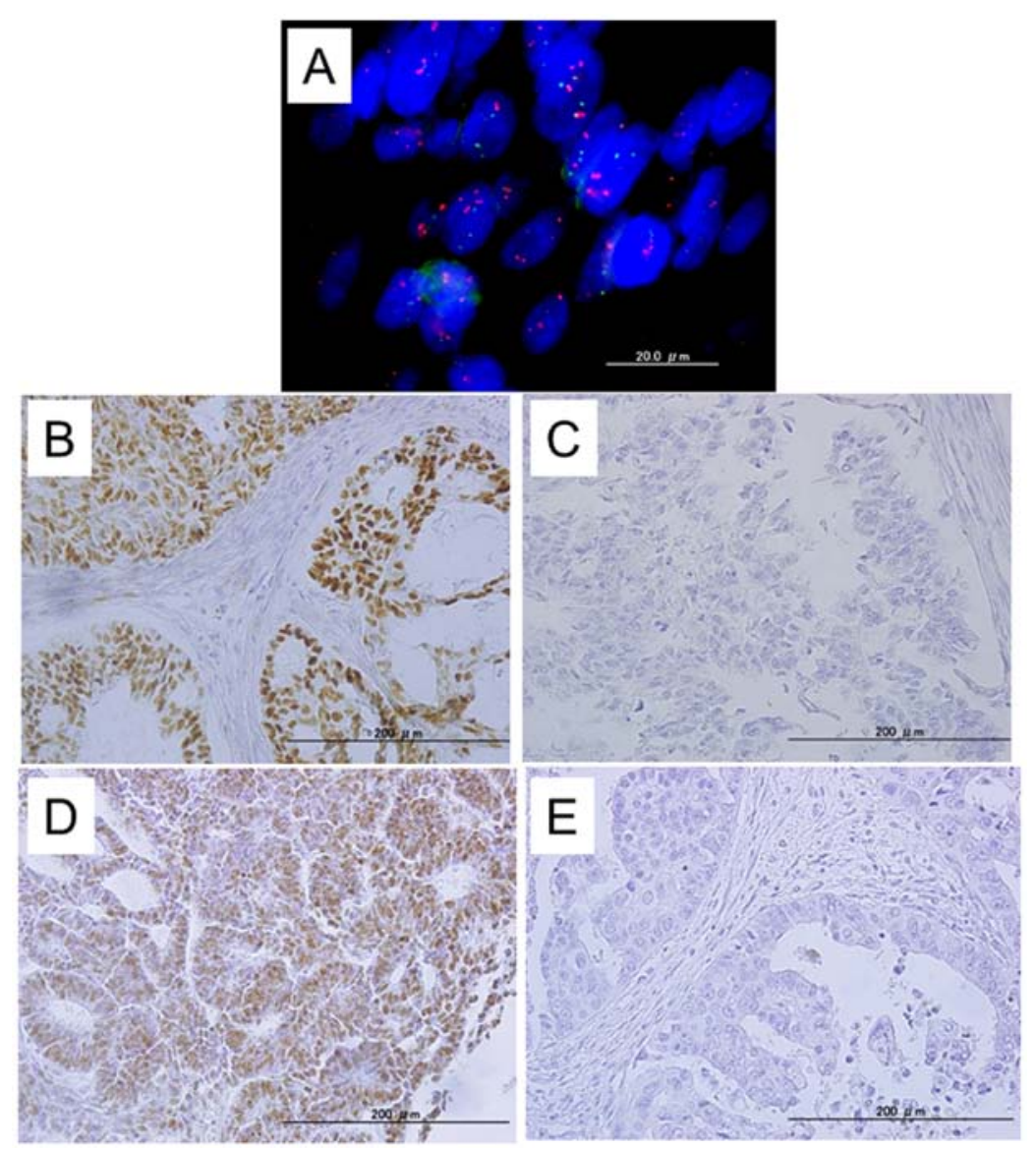

Figure 1. Dual-color fluorescence in situ hybridization (FISH) was used to detect amplification of the CCNE1 gene in endometrial endometrioid carcinomas (A) FISH analysis revealed a homogeneously stained region in the carcinoma portion with CCNE1 amplification. (B and D) A case of endometrial endometrioid carcinoma with positive immunoreactivity for CCNE1 and FBXW7 in endometrial carcinoma tissues. (C and E) A case with negative CCNE1 and FBXW7 staining in endometrial carcinoma tissues.

Table IV. Association between CCNE1 gene amplification and status of p53, hMLH1, Her2, PTEN and ARID1A in patients with endometrial cancer.

\begin{tabular}{lcccc}
\hline & & \multicolumn{2}{c}{$\begin{array}{c}\text { CCNE1 gene } \\
\text { amplification }\end{array}$} & \\
\cline { 3 - 4 } Factors & Patients & Normal & Amplification & P-value \\
\hline p53 & & & & \\
Negative & 58 & 55 & 3 & 0.2005 \\
Positive & 50 & 44 & 6 & \\
hMLH1 & & & & \\
Negative & 53 & 48 & 5 & 0.6846 \\
Positive & 55 & 51 & 4 & \\
Her2 & & & & \\
Low & 40 & 36 & 4 & 0.6308 \\
High & 68 & 63 & 5 & \\
PTEN & & & & \\
Negative & 27 & 25 & 2 & 0.041 \\
Positive & 81 & 74 & 7 & \\
ARID1A & & & & \\
Negative & 27 & 26 & 1 & 0.3149 \\
Positive & 81 & 73 & 8 & \\
\hline
\end{tabular}

CCNE1 amplification and the expression of these proteins, except for positive $\mathrm{PTEN}$ expression $(\mathrm{P}=0.041)$.

Effects of CCNE1 knockdown on endometrial endometrioid carcinoma in vitro. A panel of endometrial carcinoma cell lines was analyzed for $C C N E 1$ amplification and protein expression status (Fig. 3A). However, there were no CCNE1 amplification cell lines in the tested cells, as measured by FISH analysis (data not shown). Transfection with CCNE1 siRNA significantly reduced CCNE1 protein expression compared with control siRNA transfection (Fig. 3B). However, CCNE1 expression status correlated with the growth inhibition induced by CCNE1 siRNA (Fig. 3C). Reduction of CCNE1 expression significantly inhibited cell growth in CCNE1-overexpressing cells, including JHUC-1 and HEC108 cells. In contrast, transfection with CCNE1 siRNA did not affect cell growth in HEC251, HEC50B and HHUA cells, which exhibit moderate or minimal CCNE1 expression, suggesting that endometrial carcinomas with CCNE1 overexpression are more dependent on activation of the CCNE1-related pathway for cell proliferation and survival than those without CCNE1 expression.

\section{Discussion}

Notably, we found that there was a significantly higher frequency of $C C N E 1$ amplification in high-grade endometrial 

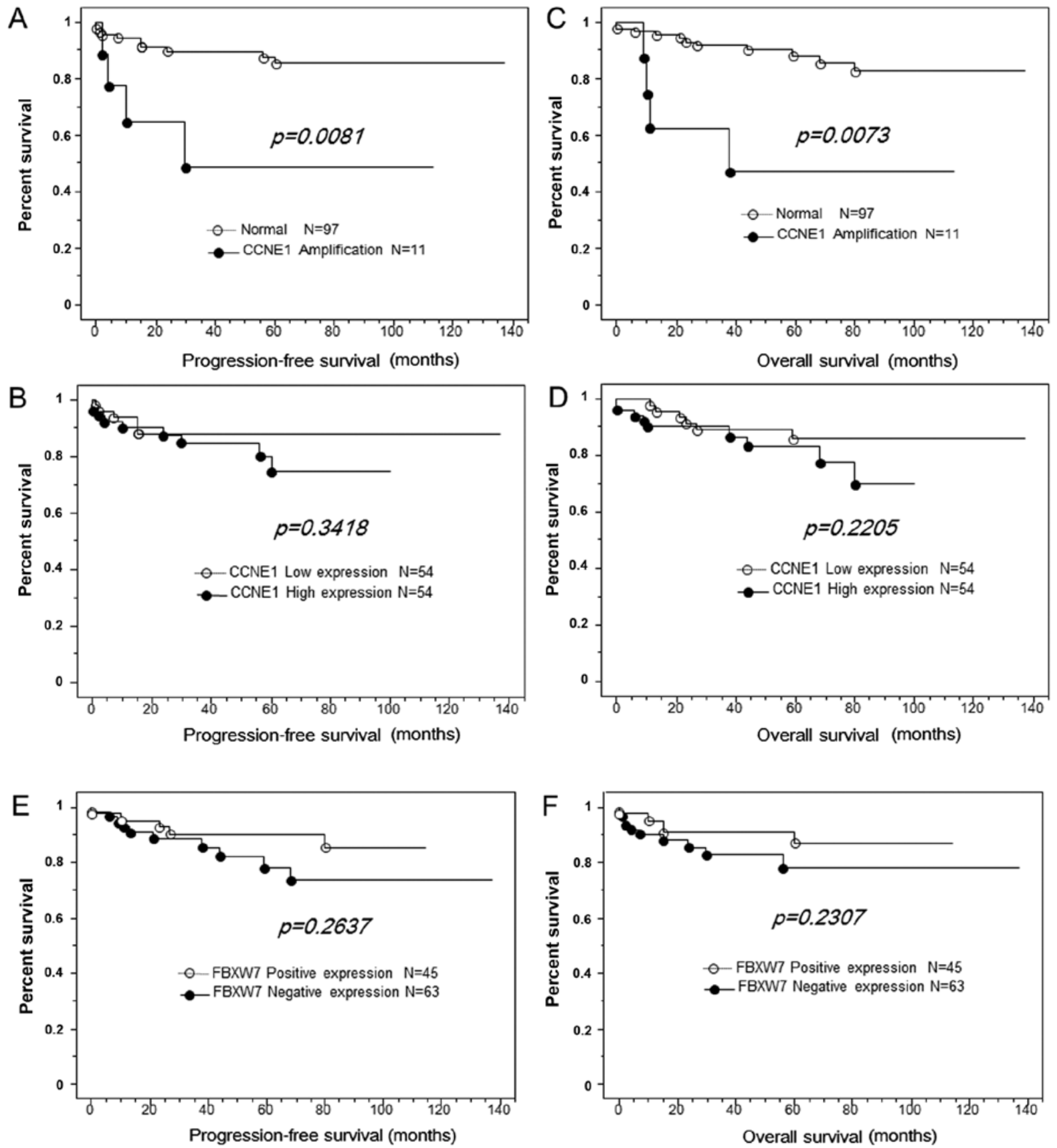

Figure 2. CCNE1 amplification is correlated with shorter progression-free/overall survival in patients with endometrial endometrioid carcinoma. (A) KaplanMeier survival analysis showed that $C C N E 1$ amplification (solid line, $\mathrm{n}=9$ ) was associated with shorter progression-free survival than absence of $C C N E 1$ amplification (dashed line, $\mathrm{n}=99)(\mathrm{P}=0.0081, \log$-rank test). CCNE1 overexpression was not significantly associated with progression-free/overall survival in patients with endometrial endometrioid carcinoma. (B) Kaplan-Meier survival analysis showed that a high CCNE1 expression (solid line, $\mathrm{n}=44$ ) was associated with shorter relapse-free survival than low CCNE1 expression; however, the difference was not statistically significant (dashed line, $\mathrm{n}=54 ; \mathrm{P}=0.3148, \log -\mathrm{rank}$ test). (C) Kaplan-Meier survival analysis showed that $C C N E 1$ amplification (solid line, $\mathrm{n}=9$ ) was associated with shorter overall survival than absence of CCNE1 amplification (dashed line, $\mathrm{n}=99 ; \mathrm{P}=0.0073$, log-rank test). (D) Kaplan-Meier survival analysis showed that high $C C N E 1$ expression (solid line, $\mathrm{n}=54$ ) was associated with shorter overall survival than low CCNE1 expression; however, this difference was not statistically significant (dashed line, $\mathrm{n}=54 ; \mathrm{P}=0.2205$, log-rank test). Negative FBXW7 expression was not significantly associated with progression-free/overall survival in patients with endometrial endometrioid carcinoma. (E) Kaplan-Meier survival analysis showed that negative FBXW7 expression (solid line, n=63) was associated with shorter progression-free survival than positive FBXW7 expression; however, this difference was not statistically significant (dashed line, $\mathrm{n}=54 ; \mathrm{P}=0.3148$, log-rank test). (F) Kaplan-Meier survival analysis showed that negative FBXW7 expression (solid line, $n=63$ ) was associated with shorter overall survival than positive FBXW7 expression; however, this difference was not statistically significant (dashed line, $\mathrm{n}=54 ; \mathrm{P}=0.2307$, log-rank test).

endometrioid carcinomas compared with that in low-grade endometrial endometrioid carcinomas. This suggested that high-grade and low-grade tumors may be distinguished based on characteristic genetic alterations. Additionally, this observation further supports the hypothesis that endo- metrial carcinoma arises from multiple pathways $(7,23)$. In this model, high-grade and low-grade carcinomas develop independently from one another and are characterized by different molecular genetic changes and gene expression profiles $(7,23)$. 
A

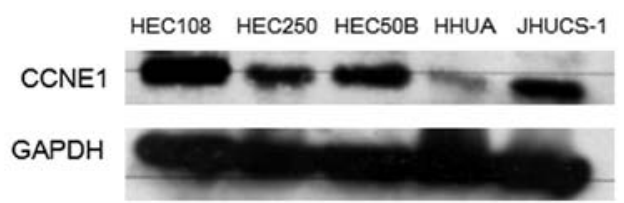

B
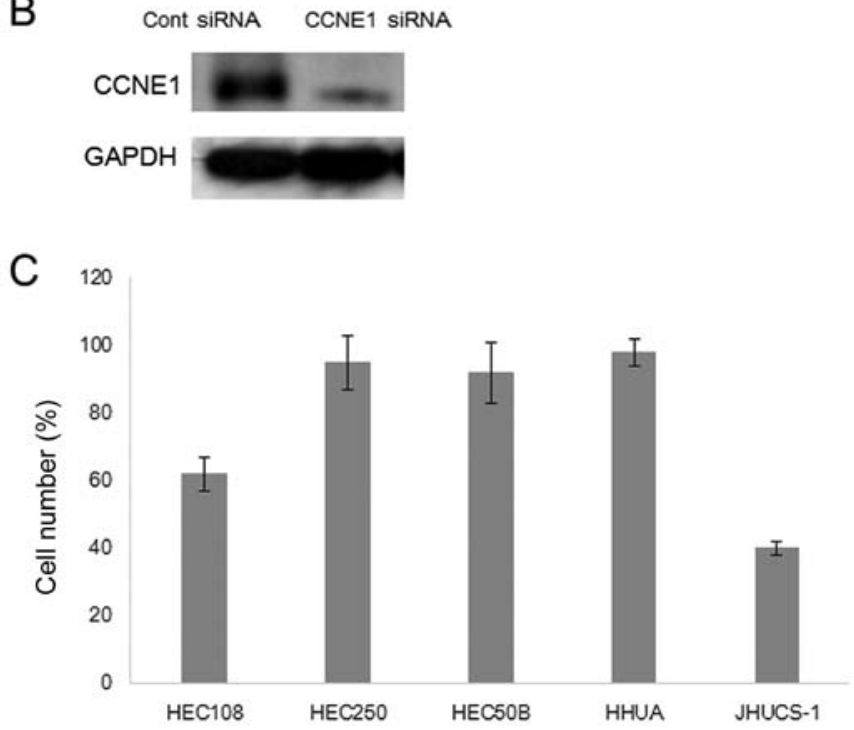

Figure 3. Western blot analysis. (A) Western blot analysis showed that CCNE1 protein expression was higher in JHUC-1 and HEC108 cells than in other cell lines. (B) Western blot analysis showed that CCNE1 protein expression was significantly decreased in CCNE1 siRNA-transfected cells compared with control siRNA-transfected cells. (C) Effects of CCNE1 siRNA on cell proliferation. Cell numbers were determined at $72 \mathrm{~h}$ after transfection with CCNE1 siRNA or control siRNA. Endometrial carcinoma cells with CCNE1 overexpression were more sensitive to growth inhibition by CCNE1 siRNA than cells without CCNE1 overexpression. The mean and SD were obtained from three experiments.

Our findings were consistent with those of a previous report showing a positive correlation between CCNE1 immunostaining and tumor grade (24). Taken together, these data, including our current FISH and immunostaining analyses, suggested that alterations in the CCNE1 gene could be a specific event in high-grade endometrial endometrioid carcinoma.

Based on our combined FISH and immunohistochemical analysis, we found that $C C N E 1$ was consistently overexpressed in all tumors exhibiting CCNE1 gene amplification (data not shown). However, some tumors overexpressed CCNE1 protein in the absence of $C C N E 1$ amplification. Therefore, we concluded that overexpression did not require gene amplification. Our findings suggested that the mechanism of CCNE1 overexpression may be dualistic, owing in part to gene amplification and in part to activation at the transcriptional level.

Bio markers that can predict clinical prognosis, including treatment response and overall survival, have substantial clinical impact on the management of patients with cancer (25). To further explore the clinical relevance of $C C N E 1$ alterations in endometrial endometrioid carcinomas, we evaluated the correlation between $C C N E 1$ amplification or protein expression and length of progression-free/overall survival in our population of patients with endometrial endometrioid carcinoma.
Interestingly, we found a strong correlation between poor prognosis and CCNE1 amplification in patients with endometrial endometrioid carcinoma. However, CCNE1 protein overexpression was associated with an insignificant trend toward poor overall survival. CCNE1 functions as a regulatory subunit of CDK2, which is essential for both the G1 to S-phase transition and DNA replication. Moreover, $\mathrm{CCNE} 1$ plays a role in apoptosis and chromosomal instability in tumor cells (26). Cyclin E-CDK2 acts through phosphorylation of substrates involved in $\mathrm{G}_{1}$ progression, $\mathrm{S}$-phase entry and centrosome duplication. This complex also has kinase-independent functions involving the loading of mini-chromosome maintenance proteins onto origins of replication as quiescent cells enter the cell cycle (27).

CCNE1 amplification has been identified as a mechanism for overexpression in endometrial carcinomas $(24,28)$. However, in the present study, we found a direct correlation between $C C N E 1$ amplification and poor outcomes, consistent with the results of another report (29). To date, CCNE1 amplification has not been shown to be explicitly associated with primary treatment resistance. The mechanism underlying the association between $C C N E 1$ amplification and shorter survival is not known; however, because mortality in patients with endometrial cancer is directly related to recurrence of disease after chemotherapy, it is conceivable that CCNE1 amplification may confer resistance to chemotherapy and/or enhance cell proliferation in chemoresistant recurrent tumors. Because the etiology of tumor recurrence is multifactorial, it is likely that $C C N E 1$ expression promotes the growth of tumor cells by providing them with higher proliferative capacity and lower apoptotic activity, as was shown in our previous study (30). Because the present study identified a previously undescribed pattern of CCNE1 amplification/expression in endometrial endometrioid carcinoma cells, further studies are required to elucidate the relationship between $C C N E 1$ gene amplification/ expression and tumor sensitivity to chemotherapy

In the present study, the absence of FBXW7 expression in endometrial endometrioid carcinoma tissue was associated with deep myometrial invasion and lymphovascular space invasion, thereby potentially affecting overall survival and progression-free survival, with marginal statistical significance. Studies have demonstrated that FBXW7 regulates a wide range of cancer-associated substrates, and FBXW7 mutation leads to high expression of CCNE1, c-MYC, Notch and MCL1. c-MYC and CCNE1 are important components of the cell cycle machinery that are frequently deregulated in cancer and possess strong oncogenic activity. FBXW7 loss and c-MYC upregulation may play roles in the aggressive biological behaviors of cancer, which may in turn induce cell proliferation (31). Cells with increased c-MYC and reduced FBXW7 expression have been shown to be more invasive and to be associated with poor prognosis due to the high levels of matrix metalloproteinases (MMP2 and MMP9) $(32,33)$. Indeed, the tendency to undergo lymph node and intrahepatic metastases may be correlated with these substrates in intrahepatic cholangiocarcinoma. Furthermore, mammalian target of rapamycin (mTOR) has been shown to function as an oncoprotein, controlling many cellular processes, such as cell proliferation and division. Interestingly, mTOR was also found to be degraded through an FBXW7-dependent 
mechanism (34). As a major regulator of a set of oncoproteins, FBXW7 has been shown to be lost and mutated in various human cancers, such as human hepatocellular carcinoma and breast carcinoma (35,36). Recently, Mitsuhashi et al (37) reported that the Notch1-JAG1 axis is related to invasive properties and a poor prognosis in patients with endometrial cancer. Peiró et al (38) reported that mTOR expression is also related to poor outcomes in endometrial cancer. Taken together, the above findings provided evidence for the mechanisms through which loss of FBXW7 expression was related to poor outcomes in endometrial endometrioid carcinoma in the present study.

Molecular genetic evidence indicates that endometrial carcinomas are likely to develop as a result of a multistep process of oncogenic activation and tumor suppressor inactivation $(39,40)$. In this study, we focused on some important genetic events of endometrial endometrioid carcinoma, i.e., inactivation of the tumor-suppressor genes PTEN, p53 and ARID1A; overexpression of the oncogene HER2; and MSI $(5,40-42)$. In addition, we also analyzed the association between PTEN, HER2, p53, MLH1 and ARID1A expression and CCNE1 amplification. No significant relationships were observed between the expression of these proteins and CCNE1 amplification, except for positive PTEN expression; these data indicated that CCNE1 amplification was independent of p53, HER2, MLH1 and ARID1A expression in endometrial endometrioid carcinoma. Notably, CCNE1 amplification and PTEN loss may occur in a mutually exclusive manner. However, assessment of mutational status via direct sequencing was not performed in this study; only immunohistochemical analysis was carried out to compare different molecular events with CCNE1 amplification status. Thus, further studies are required to fully explore the relationship between $C C N E 1$ amplification and other genetic events occurring in endometrial endometrioid carcinomas.

Cancer cells contain multiple genetic and epigenetic abnormalities. Despite this complexity, their growth and survival can often be impaired by inactivation of a single oncogene. This phenomenon, called 'oncogene addiction', provides a rationale for molecular targeted therapy (43). Based on this theory and our in vivo and in vitro findings, we propose that patients with endometrial endometrioid carcinoma having CCNE1 amplification may benefit from CDK inhibitor therapy if the disease recurs after conventional platinum and taxane or doxorubicin chemotherapy or from CDK inhibitor therapy as the primary therapy with the conventional combination of platinum and taxane or doxorubicin. To date, the CDK inhibitor E7070 has fared poorly in clinical trials for melanoma and lung cancer $(44,45)$. However, its favorable therapeutic index and high selectivity may outweigh its shortcomings in CCNE1amplified endometrial endometrioid carcinoma. Therefore, we recommend that patients participating in future clinical trials evaluating the efficacy of CDK inhibitors for endometrial endometrioid carcinoma should be stratified based on CCNE1 amplification status.

In summary, we have demonstrated that phenotypic changes in endometrial endometrioid carcinomas in response to CCNE1 inactivation depend on the expression status of CCNE1. The findings in the present study provide important insights into the biological roles of the CCNE1 signaling pathway in endome- trial endometrioid carcinomas. Additionally, our observations have important therapeutic implications for patients with endometrial endometrioid carcinoma having CCNE1 amplification. Endometrial endometrioid carcinomas with CCNE1 amplification are clinically high-grade carcinomas with aggressive behavior $(46,47)$. Therefore, detection of CCNE1 amplification in endometrial endometrioid carcinomas may identify patients who will benefit from a CCNE1-specific inhibitor.

\section{References}

1. Pisani P, Bray F and Parkin DM: Estimates of the world-wide prevalence of cancer for 25 sites in the adult population. Int $\mathbf{J}$ Cancer 97: 72-81, 2002.

2. Akhmedkhanov A, Zeleniuch-Jacquotte A and Toniolo P: Role of exogenous and endogenous hormones in endometrial cancer: Review of the evidence and research perspectives. Ann NY Acad Sci 943: 296-315, 2001.

3. Matias-Guiu X, Catasus L, Bussaglia E, Lagarda H, Garcia A, Pons C, Muñoz J, Argüelles R, Machin P and Prat J: Molecular pathology of endometrial hyperplasia and carcinoma. Hum Pathol 32: 569-577, 2001.

4. Shiozawa T and Konishi I: Early endometrial carcinoma: Clinicopathology, hormonal aspects, molecular genetics, diagnosis, and treatment. Int J Clin Oncol 11: 13-21, 2006.

5. Tashiro H, Blazes MS, Wu R, Cho KR, Bose S, Wang SI, Li J, Parsons R and Ellenson LH: Mutations in PTEN are frequent in endometrial carcinoma but rare in other common gynecological malignancies. Cancer Res 57: 3935-3940, 1997.

6. Oda K, Stokoe D, Taketani Y and McCormick F: High frequency of coexistent mutations of PIK3CA and PTEN genes in endometrial carcinoma. Cancer Res 65: 10669-10673, 2005.

7. Kuhn E, Wu RC, Guan B, Wu G, Zhang J, Wang Y, Song L, Yuan X, Wei L, Roden RB, et al: Identification of molecular pathway aberrations in uterine serous carcinoma by genomewide analyses. J Natl Cancer Inst 104: 1503-1513, 2012.

8. Welcker M and Clurman BE: FBW7 ubiquitin ligase: A tumour suppressor at the crossroads of cell division, growth and differentiation. Nat Rev Cancer 8: 83-93, 2008.

9. Spruck CH, Strohmaier H, Sangfelt O, Müller HM, Hubalek M, Müller-Holzner E, Marth C, Widschwendter M and Reed SI: hCDC4 gene mutations in endometrial cancer. Cancer Res 62: 4535-4539, 2002.

10. Knuutila S, Aalto Y, Autio K, Björkqvist AM, El-Rifai W, Hemmer S, Huhta T, Kettunen E, Kiuru-Kuhlefelt S, Larramendy ML, et al: DNA copy number losses in human neoplasms. Am J Pathol 155: 683-694, 1999.

11. Strohmaier H, Spruck CH, Kaiser P, Won KA, Sangfelt O and Reed SI: Human F-box protein hCdc4 targets cyclin E for proteolysis and is mutated in a breast cancer cell line. Nature 413: 316-322, 2001.

12. Yada M, Hatakeyama S, Kamura T, Nishiyama M, Tsunematsu R, Imaki H, Ishida N, Okumura F, Nakayama K and Nakayama KI: Phosphorylation-dependent degradation of c-Myc is mediated by the F-box protein Fbw7. EMBO J 23: 2116-2125, 2004.

13. Izumi N, Helker C, Ehling M, Behrens A, Herzog W and Adams RH: Fbxw7 controls angiogenesis by regulating endothelial Notch activity. PLoS One 7: e41116, 2012.

14. Inuzuka H, Shaik S, Onoyama I, Gao D, Tseng A, Maser RS, Zhai B, Wan L, Gutierrez A, Lau AW, et al: SCF(FBW7) regulates cellular apoptosis by targeting MCL1 for ubiquitylation and destruction. Nature 471: 104-109, 2011.

15. Yokobori T, Mimori K, Iwatsuki M, Ishii H, Onoyama I, Fukagawa T, Kuwano H, Nakayama KI and Mori M: p53-Altered FBXW7 expression determines poor prognosis in gastric cancer cases. Cancer Res 69: 3788-3794, 2009.

16. Debies MT and Welch DR: Genetic basis of human breast cancer metastasis. J Mammary Gland Biol Neoplasia 6: 441-451, 2001

17. Albertson DG, Collins C, McCormick F and Gray JW: Chromosome aberrations in solid tumors. Nat Genet 34: 369-376, 2003.

18. Wang TL, Diaz LA Jr, Romans K, Bardelli A, Saha S, Galizia G, Choti M, Donehower R, Parmigiani G, Shih IeM, et al: Digital karyotyping identifies thymidylate synthase amplification as a mechanism of resistance to 5-fluorouracil in metastatic colorectal cancer patients. Proc Natl Acad Sci USA 101: 3089-3094, 2004. 
19. Mao TL, Seidman JD, Kurman RJ and Shih IeM: Cyclin E and p16 immunoreactivity in epithelioid trophoblastic tumor--an aid in differential diagnosis. Am J Surg Pathol 30: 1105-1110, 2006.

20. Rahman M, Nakayama K, Rahman MT, Katagiri H, Katagiri A, Ishibashi T, Ishikawa M, Iida K and Miyazaki K: Clinicopathologic analysis of loss of AT-rich interactive domain 1A expression in endometrial cancer. Hum Pathol 44: 103-109, 2013.

21. Rahman MT, Nakayama K, Ishikawa M, Rahman M, Katagiri H, Katagiri A, Ishibashi T, Iida K and Miyazaki K: Fatty acid synthase is a potential therapeutic target in estrogen receptor-/ progesterone receptor-positive endometrioid endometrial cancer. Oncology 84: 166-173, 2013.

22. Nakayama K, Miyazaki K, Kanzaki A, Fukumoto M and Takebayashi Y: Expression and cisplatin sensitivity of coppertransporting P-type adenosine triphosphatase (ATP7B) in human solid carcinoma cell lines. Oncol Rep 8: 1285-1287, 2001.

23. Kandoth C, Schultz N, Cherniack AD, Akbani R, Liu Y, Shen H, Robertson AG, Pashtan I, Shen R, Benz CC, et al; Cancer Genome Atlas Research Network: Integrated genomic characterization of endometrial carcinoma. Nature 497: 67-73, 2013.

24. Kato N, Watanabe J, Jobo T, Nishimura Y, Fujisawa T, Kamata Y and Kuramoto $\mathrm{H}$ : Immunohistochemical expression of cyclin $\mathrm{E}$ in endometrial adenocarcinoma (endometrioid type) and its clinicopathological significance. J Cancer Res Clin Oncol 129: 222-226, 2003.

25. Scott $M$ and Hall PA: Prognostic and predictive factors. Methods Mol Med 97: 1-11,2004.

26. Hwang HC and Clurman BE: Cyclin E in normal and neoplastic cell cycles. Oncogene 24: 2776-2786, 2005.

27. Geng Y, Lee YM, Welcker M, Swanger J, Zagozdzon A, Winer JD, Roberts JM, Kaldis P, Clurman BE and Sicinski P: Kinase-independent function of cyclin E. Mol Cell 25: 127-139, 2007.

28. Cassia R, Moreno-Bueno G, Rodríguez-Perales S, Hardisson D, Cigudosa JC and Palacios J: Cyclin E gene (CCNE) amplification and hCDC4 mutations in endometrial carcinoma. J Pathol 201: 589-595, 2003.

29. Santala S, Talvensaari-Mattila A, Soini Y and Santala M Cyclin E expression correlates with cancer-specific survival in endometrial endometrioid adenocarcinoma. Anticancer Res 35: 3393-3397, 2015.

30. Nakayama N, Nakayama K, Shamima Y, Ishikawa M, Katagiri A, Iida K and Miyazaki K: Gene amplification CCNE1 is related to poor survival and potential therapeutic target in ovarian cancer. Cancer 116: 2621-2634, 2010 .

31. Pelengaris $S$ and Khan M: The many faces of c-MYC. Arch Biochem Biophys 416: 129-136, 2003.

32. Kubben FJ, Sier CF, van Duijn W, Griffioen G, Hanemaaijer R, van de Velde CJ, van Krieken JH, Lamers CB and Verspaget HW: Matrix metalloproteinase-2 is a consistent prognostic factor in gastric cancer. Br J Cancer 94: 1035-1040, 2006.

33. Calcagno DQ, Freitas VM, Leal MF, de Souza CR, Demachki S, Montenegro R, Assumpção PP, Khayat AS, Smith MA, dos Santos AK, et al: MYC, FBXW7 and TP53 copy number variation and expression in gastric cancer. BMC Gastroenterol 13: $141,2013$.
34. Mao JH, Kim IJ, Wu D, Climent J, Kang HC, DelRosario R and Balmain A: FBXW7 targets mTOR for degradation and cooperates with PTEN in tumor suppression. Science 321: 1499-1502, 2008.

35. Tu K, Zheng X, Zan X, Han S, Yao Y and Liu Q: Evaluation of Fbxw7 expression and its correlation with the expression of c-Myc, cyclin E and p53 in human hepatocellular carcinoma. Hepatol Res 42: 904-910, 2012.

36. Ibusuki M, Yamamoto Y, Shinriki S, Ando Y and Iwase H: Reduced expression of ubiquitin ligase FBXW7 mRNA is associated with poor prognosis in breast cancer patients. Cancer Sci 102: 439-445, 2011.

37. Mitsuhashi Y, Horiuchi A, Miyamoto T, Kashima H, Suzuki A and Shiozawa T: Prognostic significance of Notch signalling molecules and their involvement in the invasiveness of endometrial carcinoma cells. Histopathology 60: 826-837, 2012.

38. Peiró G, Peiró FM, Ortiz-Martínez F, Planelles M, SánchezTejada L, Alenda C, Ceballos S, Sánchez-Payá J and Laforga JB: Association of mammalian target of rapamycin with aggressive type II endometrial carcinomas and poor outcome: A potential target treatment. Hum Pathol 44: 218-225, 2013.

39. Okuda T, Sekizawa A, Purwosunu Y, Nagatsuka M, Morioka M, Hayashi M and Okai T: Genetics of endometrial cancers. Obstet Gynecol Int 2010: 984013, 2010.

40. Llobet D, Pallares J, Yeramian A, Santacana M, Eritja N, Velasco A, Dolcet X and Matias-Guiu X: Molecular pathology of endometrial carcinoma: Practical aspects from the diagnostic and therapeutic viewpoints. J Clin Pathol 62: 777-785, 2009.

41. Ioffe OB, Papadimitriou JC and Drachenberg CB: Correlation of proliferation indices, apoptosis, and related oncogene expression (bcl-2 and c-erbB-2) and p53 in proliferative, hyperplastic, and malignant endometrium. Hum Pathol 29: 1150-1159, 1998.

42. Sherman ME, Bur ME and Kurman RJ: p53 in endometrial cancer and its putative precursors: Evidence for diverse pathways of tumorigenesis. Hum Pathol 26: 1268-1274, 1995.

43. Weinstein IB, Joe A and Felsher D: Oncogene addiction. Cancer Res 68: 3077-3080, discussion 3080, 2008.

44. Smyth JF, Aamdal S, Awada A, Dittrich C, Caponigro F, Schöffski P, Gore M, Lesimple T, Djurasinovic N, Baron B, et al: Phase II study of E7070 in patients with metastatic melanoma. Ann Oncol 16: 158-161, 2005.

45. Talbot DC, von Pawel J, Cattell E, Yule SM, Johnston C, Zandvliet AS, Huitema AD, Norbury CJ, Ellis P, Bosquee L, et al: A randomized phase II pharmacokinetic and pharmacodynamic study of indisulam as second-line therapy in patients with advanced non-small cell lung cancer. Clin Cancer Res 13: 1816-1822, 2007.

46. Nakayama K, Nakayama N, Ishikawa M and Miyazaki K: Endometrial serous carcinoma: Its molecular characteristics and histology-specific treatment strategies. Cancers (Basel) 4: 799-807, 2012.

47. Birkeland E, Wik E, Mjøs S, Hoivik EA, Trovik J, Werner HM, Kusonmano K, Petersen K, Raeder MB, Holst F, et al: KRAS gene amplification and overexpression but not mutation associates with aggressive and metastatic endometrial cancer. $\mathrm{Br} \mathrm{J}$ Cancer 107: 1997-2004, 2012. 